\section{Dr. N. Jacob and Dr. C.O. Jacob reply}

\section{To the Editor:}

Ye and colleagues describe 2 cases of patients with seropositive rheumatoid arthritis (RA) that develop "lupus-like" symptoms under therapy with either adalimumab or infliximab. One patient developed pleuritis and presented with anti-dsDNA antibodies, but no other features of lupus. The other patient developed oral ulcers and anti-dsDNA antibodies, but no other features of lupus that were not present before the anti-tumor necrosis factor (anti-TNF) therapy.

We would emphasize the significant difference between these 2 cases and the cases described by Soforo, $e a^{1}{ }^{1}$. In those cases, full-blown active systemic lupus erythematosus (SLE) fulfilling 4 or more American College of Rheumatology criteria ${ }^{2}$ for diagnosis of SLE was demonstrated. We would hesitate to classify the 2 cases described by Ye, et al as SLE. Further, we would reiterate our cautionary directive against using TNF-blocking therapy in natural SLE.

In terms of TNF blockade, 2 classes of anti-TNF biological agents are currently licensed for clinical use: the anti-TNF monoclonal antibodies adalimumab and infliximab and a soluble TNF receptor, etanercept.

The more interesting point that Ye, et al bring up relates to whether changing a monoclonal anti-TNF agent to a soluble TNF receptor is a logical step in the management of patients with RA that develop side effects under one class of TNF blockers. We could find only a few publications in which the 3 available anti-TNF biological agents were compared in terms of their affinities, avidities, and complement activation. Avidity of binding to soluble TNF was 10 - to 20 -fold greater for etanercept than for adalimumab or infliximab ${ }^{3}$. All the anti-TNF agents bound to membrane TNF- $\alpha$ on Jurkat cell lines ${ }^{4}$. On the other hand, binding of these agents to membrane TNF in human peripheral blood mononuclear cells (PBMC) under conditions that are more similar to those in patients showed that infliximab and etanercept had lower affinities/avidities for membrane TNF than adalimumab $^{3}$. None of the 3 agents induced complement-dependent cytotoxicity when bound to activated human $\mathrm{PBMC}^{3}$. In contrast, induction of complement-dependent cytotoxicity in TNF-transfected cell lines has been reported for infliximab and adalimumab but not for etanercept ${ }^{3-5}$. Further, adalimumab or infliximab induced antibody-dependent cellular cytotoxicity (ADCC) much more potently than etanercept ${ }^{6}$. Similarly, only the monoclonal antibodies, but not etanercept, bound complement C1q in vitro ${ }^{6}$.

All 3 TNF blockers showed low-level binding to the activating complement receptors FcgRI, FcgRIIa, and FcgRIIIa, as well as to the inhibitory FcgRIIb in the absence of exogenous TNF. However, upon addition of recombinant TNF the 2 monoclonal antibodies, but not etanercept, showed increased binding to the complement receptors ${ }^{6}$. Thus there are some differences between the 2 monoclonals and etanercept in terms of complement binding, induction of complement-dependent cytotoxicity, and ADCC.

Despite the inherent limitation of each one of the assay systems used for the studies referenced here, and the unclear applicability of these studies to the situation in vivo in patients treated with these agents, it is conceivable that the differences in the ability to bind TNF and mediate cell death may account for the differences in the safety of these agents.

NOAM JACOB, MD; CHAIM O. JACOB, MD, PhD, Department of Medicine, University of Southern California Keck School of Medicine, Los Angeles, California, USA. Address correspondence to Dr. C.O. Jacob; E-mail: jacob@usc.edu

\section{REFERENCES}

1. Soforo E, Baumgartner M, Francis L, Allam F, Phillips PE, Perl A. Induction of systemic lupus erythematosus with tumor necrosis factor blockers. J Rheumatol 2010;37:204-5.

2. Tan EM, Cohen AS, Fries JF, Masi AT, McShane DJ, Rothfield NF, et al. The 1982 American College of Rheumatology revised criteria for the classification of systemic lupus erythematosus. Arthritis Rheum 1982;25:1271-7.

3. Kaymakcalan Z, Sakorafas P, Bose S, Scesney S, Xiong L, Hanzatian DK, et al. Comparison of affinities, avidities, and complement activation of adalimumab, infliximab and etanercept in binding to soluble and membrane tumor necrosis factor. Clin Immunol 2009;131:308-16.

4. Mitoma H, Horiuchi T, Tsukamoto H, Tamimoto Y, Kimoto Y, Uchino A, et al. Mechanisms for cytotoxic effects of anti-tumor necrosis factor agents on transmembrane tumor necrosis factor alpha-expressing cells: comparison among infliximab, etanercept, and adalimumab. Arthritis Rheum 2008;58:1248-57.

5. Scallon BJ, Moore MA, Trinh H, Knight DM, Ghrayeb J. Chimeric anti TNF monoclonal antibody ca2 binds recombinant transmembrane TNF-alpha and activates immune effector functions. Cytokine 1995;7:251-9.

6. Arora T, Padaki R, Liu L, Hamburger AE, Ellison AR, Stevens SR, et al. Differences in binding and effector functions between classes of TNF antagonists. Cytokine 2009;45:124-31.

J Rheumatol 2011;38:6; doi:10.3899/jrheum.101279 